\title{
Elisabeth E. Turk (ed): Forensic Pathology Reviews Vol. 6
}

\author{
Humana Press, 2011, 342 pp, hardback, ISBN 978-1-61779-248-9
}

\author{
Michael Tsokos
}

Accepted: 20 January 2012

(C) Springer Science+Business Media, LLC 2012

This sixth volume of the very successful Forensic Pathology Reviews series, now for the first time edited by Elisabeth Turk from Hamburg, Germany, fits excellently into the multinational concept of previous volumes of the series. National and international contributors in their distinct field of forensic sciences put forward the latest knowledge on special topics in 15 chapters. As in previous volumes, the book covers a broad variety of forensic pathological entities of interest for all those involved in death investigation.

The 15 chapters, illustrated with numerous color photographs, cover "Sudden natural deaths in infancy and childhood" (Langlois and Byard), "Post-mortem investigation of sudden unexpected death in infancy: role of autopsy in classification of death" (Weber and Sebire), "Sudden death from pulmonary causes" (Cunningham and Pollanen), "Sectioning of the heart, searching for pathology under the microscope" (Fineschi et al.), "Endocrine disorders with potentially fatal outcome" (Hecht), "Sudden death from infectious disease" (Morris et al.), "Aviation deaths"
(Cullen), "Fatalities in general aviation" (De Voogt), "The 9/11 attacks: the medicolegal investigation of the World Trade Center fatalities" (Gill et al.), "Injuries and fatalities in all-terrain vehicle crashes" (Mullins and Mullins), "Advances in entomological methods for death time investigation" (Villet and Amendt), "Tissue fluorescence spectroscopy in death time estimation" (Estracanholli et al.), "Heat-flow finite-element models in death time estimation" (Muggenthaler et al.), "The use of protein markers for the estimation of the post-mortem interval" (Poloz and O'Day), and "Alcohol and drug fatalities in transportation: forensictoxicological implications" (Mußhoff).

With these interesting chapter contents, the new editor has maintained the proved concept of the series.

This book makes a solid contribution to the growing number of textbooks available on forensic pathology and associated fields. Reading this book will undoubtedly be a useful experience for both beginners and experts in medicolegal and criminal investigations.

M. Tsokos ( $\square)$

Institute of Legal Medicine and Forensic Sciences,

Charité, Universitätsmedizin Berlin, Turmstr. 21 (Haus N),

10559 Berlin, Germany

e-mail: mtsokos@web.de 